\title{
Review
}

\section{Guidelines for the conduct of clinical trials for spinal cord injury as developed by the ICCP Panel: clinical trial inclusion/exclusion criteria and ethics}

MH Tuszynski*,1,2 ${ }^{\text {JD Steeves }}{ }^{3}$, JW Fawcett ${ }^{4}$, D Lammertse ${ }^{5,6}$, M Kalichman ${ }^{7}$, C Rask $^{8}$, A Curt ${ }^{2}$, JF Ditunno ${ }^{9}$, MG Fehlings ${ }^{10}$, JD Guest ${ }^{11}$, PH Ellaway ${ }^{12}$, N Kleitman ${ }^{13}$, PF Bartlett ${ }^{14}$, AR Blight ${ }^{15}$, V Dietz ${ }^{16}$, BH Dobkin ${ }^{17}$, R Grossman ${ }^{18}$ and A Privat ${ }^{19}$

${ }^{1}$ Department of Neurosciences, Center for Neural Repair, University of California,-San Diego, La Jolla, CA, USA; ${ }^{2}$ Department of Neurology, Veterans Administration Medical Center, La Jolla, CA, USA; ${ }^{3}$ ICORD, University of British Columbia and Vancouver Coastal Health Research Institute, Vancouver, British Columbia, Canada; ${ }^{4}$ Cambridge University Centre for Brain Repair, Robinson Way, Cambridge, UK; ${ }^{5}$ Craig Hospital, Englewood, CO, USA;

${ }^{6}$ Department of Physical Medicine and Rehabilitation, University of Colorado at Denver and Health Sciences Center, Denver, CO, USA; ${ }^{7}$ Research Ethics Program and Department of Pathology, University of California - San Diego, La Jolla, CA, USA; ${ }^{8}$ Institute for OneWorld Health, San Francisco, CA, USA; 9 Department of Rehabilitation Medicine, Jefferson Medical College of Thomas Jefferson University, Philadelphia, PA, USA; ${ }^{10}$ Department of Surgery, University of Toronto, Division of Neurosurgery, Toronto, Ontario, Canada; ${ }^{11}$ Department of Neurosurgery, University of Miami, Miami, FL, USA; ${ }^{12}$ Department of Movement \& Balance, Division of Neuroscience \& Mental Health, Imperial College London, London, UK, ${ }^{13}$ National Institute of Neurological Disorders and Stroke, National Institutes of Health, Bethesda, MD, USA; ${ }^{14}$ National Institute of Neurological Disorders and Stroke, Bethesda, MD, USA; ${ }^{15}$ Acorda Therapeutics, Inc., Hawthorne, New York, NY, USA; ${ }^{16}$ Spinal Cord Injury Center, Balgrist University Hospital, Zurich, Switzerland; ${ }^{17}$ Department of Neurology, University of California Los Angeles, Geffen School of Medicine, Neurologic Rehabilitation and Research Program, Los Angeles, CA, USA; ${ }^{18}$ Methodist Neurological Institute, Houston, TX, USA; ${ }^{19}$ Institut des Neurosciences - CHU St Eloi, INSERM U-583, Montpellier Cedex, France

The International Campaign for Cures of Spinal Cord Injury Paralysis established a panel tasked with reviewing the methodology for clinical trials for spinal cord injury (SCI), and making recommendations on the conduct of future trials. This is the third of four papers. It examines inclusion and exclusion criteria that can influence the design and analysis of clinical trials in SCI, together with confounding variables and ethical considerations. Inclusion and exclusion criteria for clinical trials should consider several factors. Among these are (1) the enrollment of subjects at appropriate stages after SCI, where there is supporting data from animal models or previous human studies; (2) the severity, level, type, or size of the cord injury, which can influence spontaneous recovery rate and likelihood that an experimental treatment will clinically benefit the subject; and (3) the confounding effects of various independent variables such as pre-existing or concomitant medical conditions, other medications, surgical interventions, and rehabilitation regimens. An issue of substantial importance in the design of clinical trials for SCI is the inclusion of blinded assessments and sham surgery controls: every effort should be made to address these major issues prospectively and carefully, if clear and objective information is to be gained from a clinical trial. The highest ethical standards must be respected in the performance of clinical trials, including the adequacy and clarity of informed consent.

Spinal Cord (2007) 45, 222-231. doi:10.1038/sj.sc.3102009; published online 19 December 2006

Keywords: spinal cord injury; clinical trial; clinical assessment; confounding variables; inclusion criteria; exclusion criteria; ethics; informed consent

*Correspondence: MH Tuszynski, Department of Neurosciences, Center for Neural Repair, University of California- San Diego, La Jolla, CA 92093, USA

\section{Introduction}

This is the third paper of a series of four, reporting the deliberations of a panel sponsored by the International 
Campaign for Cures of spinal cord injury Paralysis (ICCP), an affiliation of 'not for profit' organizations, one of whose missions is to facilitate the translation of valid treatments for spinal cord injury (SCI) paralysis.

The four papers address considerations relevant to the planning and design of therapeutic trials in SCI. Their subjects are:

1. natural history of SCI, degree and time course of spontaneous recovery, and statistical power needed to achieve a valid outcome;

2. appropriate clinical outcome measures for different clinical phases and targets;

3. patient selection criteria (inclusion/exclusion), confounding variables, and ethics; and

4. trial design, statistical analysis, and organization of multicenter trials.

This third paper from the ICCP Clinical Guidelines Panel addresses the topic of inclusion/exclusion criteria, confounding variables, and ethics.

The design of clinical trials in SCI must take into consideration a number of factors that can, at the end of the trial, significantly impact the ability to detect the potential benefit of an experimental substance and yield useful information. Given the limited pool of individuals with SCI, the need to design rational trials is particularly pressing. Worldwide, it is believed that approximately 150-200 000 people suffer acute SCI each year, with 2.5 million individuals living in chronic stages of injury. The approximate distribution of cases in some representative countries is as follows:

\begin{tabular}{lcc}
\hline Country & $\begin{array}{c}\text { Number of acute } \\
\text { SCI cases/year }\end{array}$ & $\begin{array}{c}\text { Number of } \\
\text { chronic SCI cases }\end{array}$ \\
\hline United States & 11000 & 280000 \\
Canada & 1200 & 40000 \\
France & 1200 & 40000 \\
United Kingdom & 800 & 30000 \\
Germany & 1500 & 50000 \\
\hline
\end{tabular}

The following sections provide information regarding a number of variables that can affect the design, enrollment, and analysis of all phases of a clinical trial. The purpose of a Phase 1 clinical trial is primarily to assess safety and to identify the maximum tolerated or feasible dose of a drug. Phase 2 studies begin to evaluate appropriate clinical efficacy measures, and provide information on dosing as well as the potential size and variability of the potential treatment effect in preparation for definitive Phase 3 trials. Optimally, Phase 3 trials are prospective, randomized, placebo- or shamcontrolled studies with clearly defined primary and secondary efficacy end points that can lead to regulatory approval for an experimental therapy (for further discussion see Lammertse et $a l^{1}$ ).

\section{Inclusion criteria in SCI clinical trials}

\section{Timeframe of subject enrollment}

Clinical trial designs should take into consideration the mechanism of the candidate therapy, and thereby target appropriate time points postinjury.

For example, a candidate neuroprotective therapy would target early stages of spinal cord injury, typically requiring application of the experimental therapeutic within hours to days of injury. In general, secondary damage after SCI is believed to significantly worsen long-term disability, and it begins within minutes of injury and may continue for several weeks. Some neuroprotective therapies target specific cellular components that contribute to secondary injury, such as inflammatory cells, free radicals, excitatory molecules, or intracellular signalling cascades. Given the differing nature of these potential targets, and the different potential time frames for achieving a beneficial effect for each therapeutic, the specific time frame for clinical application should be established in preclinical animal studies.

Other potential therapies may promote repair (eg axonal regeneration) or plasticity (eg changes in synaptic connectivity). Such therapies may be relevant to acute, subacute, or chronic stages of SCI. Whichever stage of injury is targeted, it should be supported by preclinical animal data indicating efficacy at the corresponding postinjury time range to be clinically tested. It should also be noted that the chronic stage of injury will provide the most static functional baseline, because minimal spontaneous neurological improvement occurs after an individual has survived with SCI for a chronic period of time. As a result, a chronic trial requires a smaller number of subjects to be enrolled and has the greatest statistical power for detecting potentially significant treatment effects in a clinical trial (see below and Fawcett et $a l^{2}$ ). However, the chronic injury period is potentially the most difficult time point to biologically influence the injured spinal cord in a beneficial manner. Thus, it is recommended that the postinjury human subject recruitment period for a clinical trial closely mimic indices of efficacy emerging from preceding preclinical animal models, to enhance the probability of detecting clinical benefit. For further discussion of the various stages of SCI, see the accompanying paper SCI Trial Guidelines $1 .^{2}$

\section{Enrollment of subjects with complete vs incomplete injuries}

As noted in SCI Trial Guidelines $1,{ }^{2}$ the natural history of recovery markedly differs in subjects with clinically complete SCI compared with subjects with clinically incomplete injuries. Further, the rate of spontaneous improvement is greatest within the first 3 months after injury, therefore the ability to predict spontaneous improvement in function improves as the length of time after the injury increases. Among subjects examined and classified with the ASIA Impairment Scale within $72 \mathrm{~h}$ of 
SCI, roughly $20 \%$ of clinically complete patients (ASIA A) show spontaneous functional improvement by 1 year postinjury, whereas $50 \%$ or more of patients with incomplete functional impairment (ASIA B or ASIA C) show spontaneous functional improvement $(>50 \%$ of ASIA B subjects improve at least one grade, and $\sim 75 \%$ of ASIA C patients improve to ASIA D). Thus, any therapy initiated within $72 \mathrm{~h}$ of injury (acute stage of injury) will need to include a very large number of subjects to detect a significant difference between treatment and control groups, if patients with clinically incomplete injuries are enrolled in the study (for sample power analyses, see Fawcett et $a l^{2}$ ). A study enrolling only ASIA A subjects will show less spontaneous improvement by the control group over time, allowing enrollment of fewer subjects to detect potentially statistically significant outcomes. On the other hand, a therapy that acts by enhancing plasticity of spared central pathways may be less likely to exert a detectable effect in patients with clinically complete injuries. The design of a trial must carefully consider these possibilities to avoid exposing patients to undue risk with little expectation of benefit, and to enhance the likelihood that useful information will be forthcoming.

At subacute stages of injury, approximately $10 \%$ of clinically complete patients (ASIA A) will subsequently show some spontaneous functional improvement by one year postinjury, whereas approximately $40-50 \%$ of patients with incomplete injuries (ASIA B and ASIA C) continue to exhibit spontaneous functional improvement by one year. Thus, a therapy directed at this stage after injury will show slightly more stability in terms of spontaneous recovery by control subjects than an acute SCI study, particularly if it restricts enrollment to clinically complete patients. As a consequence, a subacute study could enroll slightly fewer patients to detect a potentially significant outcome benefit compared to an acute therapy. ${ }^{2}$

At much later stages of injury (subjects examined 6-12 months postinjury), most studies suggest that fewer than $5 \%$ of clinically complete patients (ASIA A) continue to show spontaneous functional improvement by one year postinjury, whereas $10-20 \%$ of patients with incomplete injuries (ASIA B and ASIA C) may continue to exhibit further spontaneous improvement. Thus, a therapy directed at subjects with chronic injury will be able to detect a potential effect of treatment with a much smaller cohort of patients than acute and subacute injury trials. ${ }^{2}$

If clinical trials enroll subject groups that span acute, subacute, or chronic stages of injury, it may be worthwhile to stratify these groups for purposes of statistical analysis. Group stratification would allow identification of particular stages of injury that are most responsive to an experimental treatment. It should also be noted, however, that stratification decreases the overall power of a study. Thus, it is important to prospectively identify the most important comparisons for statistical analysis, and to consult an experienced statistician when planning a trial.
Enrollment of subjects at various levels of injury (cervical, thoracic, lumbar)

Depending on the nature of a particular experimental therapy, it will be important to take into consideration the level of SCI in the design of the clinical trial. Factors such as mechanism of drug or cell effect, route of administration, distance of spinal segments over which the therapeutic agent is likely to act, and the potential risks of the therapy can influence the choice of injury level for the study.

Therapeutics acting over only short distances may be likely to improve functional outcomes only at spinal cord levels immediately adjacent to the injury site. For example, a cell graft into the lesion site that will support axon growth or remyelination for only short distances may be more likely to demonstrate clinical benefit if placed in cervical, lumbar, or low thoracic spinal cord segments, thereby excluding subjects with mid- or highthoracic injuries. In the former cases, denervated target neurons would be located immediately adjacent to the implant, and thus would more readily provide evidence of reinnervation. Placing a cell graft in a mid- or highthoracic segment might be less likely to affect an observable functional outcome, unless preclinical animal studies demonstrated axon growth over very long distances (eg $\sim 10 \mathrm{~cm}$; something yet to be convincingly reported in the preclinical literature) or transmission of neural activity to distant segments by propagation via interneuronal relays (propriospinal circuits).

On the other hand, the greatest risk for adverse effects would come from manipulation of cervical and lumbar spinal cord regions, if critical spared spinal cord tissue rostral to the lesion site is compromised. Work is in progress, therefore, to develop clinical assessment tools that would detect benefits and risks of even shortdistance axon growth within the thoracic spinal cord, ${ }^{3,4}$ as described in the preceding article. ${ }^{5}$ However, such tools are not yet commonly available.

Hence, a potential trial should weigh the benefits and risks of treating cervical or lumbar versus thoracic segments. In the initiation of a clinical trial of a therapeutic, which is thought to act over only a short distance, it might be reasonable to first establish safety in several subjects with thoracic levels of SCI, and then extend the trial to subjects with cervical or lumbar levels of injury after safety of the intervention has been established.

For therapies that are predicted to extend over many spinal segments, thoracic injured subjects may be better candidates for enrollment. For example, therapeutics that could enhance plasticity of spared systems, such as myelin neutralization or administration of growth factors, could have effects at lumbar segments even when administered at thoracic levels; this could lead to functional neuronal sprouting (plasticity) within lumbar segments, hypothetically contributing to functional recovery. A clinical trial of such an agent, after showing earlier safety in thoracic-injured subjects, might then proceed to enroll SCI subjects with all levels of injury. 
Therapies that may cause functional deterioration within spinal segments rostral to the spinal lesion site require the greatest caution in transition to human testing. Phase 1 trials of such approaches should provide clear evidence of safety and tolerability in subjects with thoracic-level injuries before moving to cervical or lumbar segments.

\section{Age}

Most clinical trials in SCI have limited the age range of subject enrollment to 15 or 16 years at the lower limit, and 55-70 years at upper limits. Physiological and ethical considerations should be taken into consideration when selecting age guidelines. These considerations include the possibility that very young subjects are likely to have enhanced spontaneous plasticity, and aged subjects may exhibit diminished spontaneous plasticity. Both of these extremes would increase the variability of outcome in a clinical trial, and would therefore represent a confounding influence. Although consensus is lacking in the field of neural plasticity and repair, it is generally believed that enhanced plasticity persists up until 2 years of age, and possibly longer. Similarly, the limited amount of evidence available in the scientific and clinical literature suggests that plasticity or spontaneous recovery from central nervous system (CNS) damage may be diminished above the age of 65 or 70 .

The informed consent procedure is an ethical challenge for subjects under 18 years of age. Therapies entailing greater risk represent proportionately greater ethical challenges to obtaining informed consent. Consent of parents or primary caregivers/guardians is necessary for an individual younger than the legal age of consent, or in individuals with impaired cognitive capacity. It is further noted that children provide 'assent' but not 'consent' for clinical trials. Thus, it is imperative to be aware of the possibility that parents or legal guardians of children with SCI may have a reduced capacity to provide truly informed consent, or be expected to show their best judgment when a child in their care has recently suffered a spinal injury.

Aging is often accompanied by coexisting medical conditions that may influence CNS plasticity or functional recovery. These potentially confounding factors include diabetes, elevated blood pressure, musculoskeletal disorders, use of concomitant medications or postinjury rehabilitation, all of which may affect plasticity and CNS function. Further, the pathophysiology of SCI may change with age. Although fracturedislocation injuries constitute a substantial proportion of injuries in younger adults, degenerative disc or spine disease becomes an increasingly frequent mechanism of injury in aged individuals. Aging also contributes to a risk of additional spinal pathology at a level other than the injury, including spinal stenosis and nerve root pathology at lumbar or cervical levels, as well as an increased incidence of peripheral neuropathy. These factors should be considered in trial design.

\section{Lesion anatomy considerations}

Modern imaging techniques provide the opportunity to characterize lesions at the time of treatment to support subsequent outcome analysis in the trial. ${ }^{5}$ Thin-plane sagittal and axial MRI imaging through a lesion site can provide data regarding lesion location, size, associated edema, parenchymal sparing, and the extent of hemorrhage. A possibility exists to stratify analysis by the nature of the lesion on MRI. Such analyses could enhance matching of enrolled subjects into treatment and control groups, supporting exploratory effect analyses in Phase 1 and 2 trials.

MRI studies of SCI have shown that the majority of lesions are focal within one or two spinal segments. A small subset of subjects has two or more lesions at different spinal cord levels after trauma. Other patients, including those with underlying congenital or acquired spinal stenosis, have evidence of more complex injuries that are contiguous over several spinal segments. These latter two groups should be excluded from early SCI trials owing to the likelihood that multiple levels of spinal injury will confound the interpretation of any clinical outcome. Concomitant brain injury can also confound the ability of a participant to provide informed consent, comply with the trial protocol, or respond accurately during study assessments. For these and other reasons, people with concomitant brain and spinal injuries should be excluded from enrollment in a trial.

Trials of therapies aiming to promote the plasticity (particularly axonal sprouting) of spared neuronal pathways should consider excluding individuals with complete anatomical transection. There is also some evidence that the underlying pathological substrates of gunshot wounds to the spine may differ fundamentally from contusive damage. That is, gunshot wounds are a relatively inhomogeneous form of injury, reflecting the effects of lacerating trauma, as well as force/indirect/ contusive mechanisms. Thus, outcomes may be more heterogeneous after gunshot injury than contusion injury, representing a potentially confounding variable.

Some individuals develop spinal cord damage from non-traumatic causes, including transverse myelitis, multiple sclerosis, cancer, abscess, or vascular occlusion. The pathophysiology of these processes is likely to differ sufficiently from traumatic injury that such individuals should not be recruited to SCI studies that are primarily enrolling acute compressive/contusive SCI.

\section{Rehabilitation}

There is a possibility that programs of physical rehabilitation constitute independent (confounding) variables for any outcome after SCI. Augmented physical therapy may enhance plasticity and improve some features of functional clinical end points, whereas inactivity may worsen recovery. It is also possible in chronic patients that rehabilitation before experimental treatment could influence trial outcomes. Study design should consider standardizing or stratifying rehabilitation to the extent that this is possible. ${ }^{1,5}$ 
Nature of acute/subacute medical care

A number of issues related to 'standard medical care,' which fall outside the scope of an experimental therapy for SCI, could constitute confounding factors in trial outcome. These factors should be considered and addressed, when possible. ${ }^{1,5}$ The following is a partial list of potential confounding variables related to 'standard medical care':

1. Practices regarding the timing and nature of postinjury surgical procedures vary extensively across medical centers. For example, early post-traumatic spinal decompression is routinely performed in some centers, is delayed in others, or may not be performed at all in other centers. ${ }^{6}$ Similarly, cervical spine traction is used in some cases of SCI but not in others. ${ }^{7}$ Moreover, the extent and completeness of spinal cord decompression may be an important covariate that would need to be tracked (controlled for) in clinical trial planning. Detethering of the chronically injured spinal cord in clinically stable patients is another practice lacking clear consensus regarding the short- or long-term functional benefits.

2. The use of high-dose methylprednisolone is routine in some treatment centers, whereas it is only used occasionally or not at all in others.

3. Standard targets for blood pressure management may vary between institutions.

4. Antibiotics are routinely used to treat or prevent infections in some SCI patients, and the choice of antibiotic may influence outcome. For example, minocycline, an antibiotic of the tetracycline family, may itself be neuroprotective and is currently the subject of a prospective clinical trial for SCI.

5. Altered body temperature, hemoglobin level, and any concurrent infection causing systemic elevation of inflammatory cells and cytokines also represent independent variables that may influence outcome after SCI.

The sheer number of unknown effects for these and other potential confounding factors on outcomes after SCI make it extremely difficult to prospectively control all factors when designing a study. In larger trials, the process of randomization may equalize some of these confounding factors across treatment groups. However, we recommend that attempts be made when screening potential trial sites to track institutional practices regarding standards of medical care and identify and track potential confounding variables.

When possible, confounding variables should be normalized by the study designers. For example, the use of potentially confounding drugs, such as antiinflammatory medications and tetracycline-class antibiotics, may be proscribed in the trial design, with exceptions allowed for necessary medical treatment. Concurrent use or omission of steroid therapy in a trial can be mandated by study investigators. The timing and nature of surgical therapy can be proscribed by study designers in 'routine' cases of SCI that do not pose special cases of medical or surgical management. All concomitant illnesses, vital signs, and treatments should be recorded for the purpose of review at the conclusion of the trial.

\section{Other considerations}

For entry into most SCI clinical trials, subjects should not have other diseases that are expected to interfere with the study (eg, dementia, autoimmune disease, preexisting demyelinating disease). Although gender may be a confounding factor in an SCI trial owing to potential effects of estrogen-related systems on outcomes from nervous system injury, previous trials have not identified gender as a confounding influence and it may not be practical or justifiable to limit study enrollment based on gender. Females of reproductive age should be screened for pregnancy prior to enrollment and agree to use appropriate double-barrier contraceptive methods to avoid pregnancy for the duration of a study.

\section{Exclusion criteria in SCI clinical trials}

Concomitant medications or treatments

Certain classes of drugs may influence outcome from SCI. These should be recorded during the trial, and consideration given to post hoc analysis controlling for the use of these drugs.

As noted above, there are no clear standards for the use of steroids such as methylprednisolone after SCI. Different trials in the future may choose to include or exclude steroid use. If proscriptive criteria regarding steroid use are not included in a trial, then there should be an attempt to control for steroid use. This can be done by matching the number of treated and control patients with regard to steroid use, or prospectively planning sub-analyses based on steroid use. The latter approach, however, may reduce statistical power and impractically elevate the required number of subjects enrolled in a trial.

Until more is known regarding the role of tetracycline-class antibiotics in influencing recovery after SCI, study designers should consider excluding or controlling for enrollment of patients actively using these drugs because they may constitute independent variables affecting study outcome. Similarly, use of GM-1 ganglioside (Sygen) should not be allowed in an uncontrolled manner. Other classes of drugs hypothetically could also affect outcome after SCI, including statins, valproic acid, steroids (not limited to methylprednisolone), anti-inflammatory drugs, antispasmodics, erythropoietin, and botulinum toxin. Ideally, experiments in animal models would test potential interactions of an experimental treatment with potentially confounding drugs. Minimally, all concomitant medications must be recorded in a clinical trial. Alternative interventions used after injury should also be noted, such as acupuncture, herbal medicines, or 'homeopathic' approaches. 
Previous participation in an experimental trial for SCI would constitute a potentially confounding variable in the interpretation of efficacy or safety of the proposed intervention. Such previous trials could include either medications or implanted cells. Other interventions could also affect trial outcome, including tendon transfers, implanted devices (bladder stimulation, chronic intrathecal infusions), or permanent modifications of anatomy (dorsal rhizotomy). It is noted that chronic SCI clinical trials may provide an opportunity to control for some of the preceding variables, for example, concomitant drug use, and to reduce potential effects of these independent variables.

\section{Pre-existing medical conditions}

As noted previously, some premorbid diseases should exclude enrollment in a therapeutic trial for SCI, particularly those affecting the nervous system. These include demyelinating disease, previous SCI, or other neurological system illnesses and significant antecedent trauma. Systemic plasticity-modifying agents could influence risk of seizures.

Chronic medical conditions such as diabetes, hypertension, autoimmune disease, and psychiatric illness may also influence trial outcome. Whether trials should limit subject enrollment owing to these factors should be decided in individual studies, based upon the suspected mechanism of the therapeutic agent.

\section{Anatomical considerations}

The state of the spinal cord caudal or rostral to an injury site may influence outcome. For example, syringomyelia, spinal stenosis, plexopathy, neuropathy, radiculopathy, cauda equina injury, myelitis, demyelination, ischemia, multiple spinal cord injuries, severe spinal degenerative pathologies, or ongoing focal spinal cord compression may influence outcome and could be considered as exclusionary factors in certain trials. Individuals with anatomically complete lesions should not be enrolled in trials of therapeutics that act solely by influencing spared axonal projections and their potential connectivity with target cells.

\section{Ability to collect data}

An inability to perform accurate and complete baseline examinations may constitute an exclusionary criterion for trial participation. This could arise in the case of concomitant head trauma, unreliable communication (eg, foreign language or aphasia), intoxication, intubation, sedation, or immobilization for treatment of an extremity injury. A psychotic disorder or untreated premorbid major depression may also affect ability to complete a trial (compliance) or collect data. Ongoing alcohol or substance abuse may either impair ability to participate reliably in a trial, or may constitute a confounding factor if withdrawal occurs during a trial.

\section{Ethics}

A study involving risk to human subjects cannot be ethically defensible if it is not scientifically defensible. The other components of this series of documents attempt to identify salient issues surrounding the design of solid, interpretable trials; a number of additional ethical considerations arise in the conduct of SCI clinical trials and are discussed in this section.

\section{Sham surgery controls}

Individual, cultural, statistical, and regulatory considerations impact the inclusion and the nature of control subjects in SCI clinical trials. It is generally agreed that the clearest and most reliable information regarding the actual value of a potential therapy requires the analysis of some type of control group, and the masking or blinding of the subject and examiner to the identity of the treatment group. Further, it is possible that a simple surgical procedure itself, independent of any injected test substance or cell, may improve outcome after SCI. This could be a consequence of surgical decompression, detethering, improved cerebrospinal fluid flow dynamics around the lesion site, or other factors, including the potentially powerful effects of subject and investigator expectation about desired outcomes and benefits.

The choice of an appropriately matched control group, and the ability to perform double-blinded analyses, is not always a straightforward issue in SCI. For example, there is little doubt regarding the inclusion of a control group when the substance under investigation is an orally administered drug with a relatively safe adverse event profile. In this case, a placebo control arm is simple to include in the study, and double blinding can be implemented.

However, when the study drug requires open surgical manipulation, then the use of a sham surgery 'control' group may subject a patient to risk of adverse events caused by the sham procedure itself. SCI patients may be medically unstable, concurrently infected, or at high risk of suffering postoperative complications such as pneumonia or other infection. Sham surgical procedures could also lead to autonomic dysreflexia. These risks are not trivial, and are particularly notable after acute SCI.

However, these risks may be mitigated by the value to be gained from the ability of science and medicine to conduct a clinical trial that will lead to a statistically interpretable outcome. ${ }^{8,9}$ Under these circumstances, an experimental SCI trial could be of benefit to society. Discussions in the medical literature generally support the inclusion of control groups in clinical trials, even when these control procedures could represent some risk to the subject's health. ${ }^{9}$ Indeed, the consequences to humanity of allowing invasive surgical procedures without the adequate study of control groups has, in other disease states, led to countless unnecessary surgeries and their coexistent morbidities. Examples of this include mammillary artery bypass for ischemic heart disease and extracranial-to-intracranial artery bypass 
procedures for cerebral ischemia. ${ }^{10}$ There is a real risk that a surgical intervention without real benefit for SCI could gain wide acceptance and implementation owing to the lack of performance of a clearly interpretable clinical trial. In such a case, hundreds of thousands of SCI patients could subsequently be exposed to unnecessary surgical procedures that in some proportion of patients would lead to medical complications, potentially worsening their disability or leading to death.

In spite of the great potential benefit of such studies, it is not a sufficient argument to justify the possible assignment of research subjects to a sham surgery group. Even though the research investigators and ethics review committees might conclude that such research is acceptable, it ultimately must be up to the individual research subject to decide whether he or she perceives their personal risks to be justified by the possible benefits to society. The burden is on investigators to ensure that potential subjects will only be enrolled once they clearly understand and accept the possibility of assignment to the control group, the uncertainty of any possible benefit, and the nature of likely and possible risks of participation in the research study. This is a minimal expectation for any research study, but the standards for informed consent must be higher than normal for a project that requires sham surgery on the CNS.

A precedent for the inclusion of sham surgical controls has already been established in Parkinson's disease. ${ }^{11,12}$ Two prospective, randomized, multicenter, placebo-controlled trials of fetal grafting for Parkinson's disease employed sham surgery control groups who underwent scalp incision and partial burr hole, without penetration of the dura mater. Notably, results of both studies showed no statistically significant difference in outcome between the treated and control arms of the study.

Given the desirability of including a valid control group in any Phase 2 or 3 human clinical trial, there are various ways in which these control groups could be designed into SCI trials. First, a parallel, nonoperated group could be enrolled into a trial and assessed in a parallel fashion to a treatment group. The characteristics of a nonoperated control group should match, as closely as possible, the treatment group with regard to age and gender, and in relation to injury level, severity and, time since SCI. Although a non-surgical control group of patients could not be blinded, their examiners should be blinded, allowing at least a single-blinded assessment of study outcome.

A hypothetically ideal sham surgery group would consist of subjects undergoing the same surgical procedure as the control group, up to the point of partial spinal laminectomy. For reasons stated above, this type of invasive control group may not be universally acceptable to all SCI trial investigators. However, many patients routinely undergo acute spinal decompression after SCI as part of 'standard' postinjury care; thus, an experimental intervention delivered during a surgical procedure, which would in any event be conducted as part of 'standard medical care', would provide the opportunity for creation of a sham group and, therefore, double blinding. If test substance administration is not possible during a surgical procedure already planned as part of 'standard medical therapy', then sham controls consisting of skin incision only under anesthesia, or skin incision plus subcutaneous dissection, can also be considered. To minimize the risk of either experimental SCI therapy or sham control procedures, it is desirable to develop less invasive surgical techniques. Image guided and minimally invasive surgical approaches are being explored in this regard. These last two surgical control options might constitute a relatively low and acceptable risk to an SCI patient and, as pointed out above, would be of significant potential benefit to society because such a randomized controlled trial would provide the best opportunity for obtaining clearly interpretable results.

If for some reason an SCI trial does not include a sham- or placebo-control group, then investigators should enroll a concurrent 'standard of care' treatment group that is matched to the experimental group for patient demographics and injury characteristics, as stated previously. In chronic SCI studies, participants could be compared with their stable pretreatment baseline, but a parallel sham surgery, placebo or concurrent no-treatment group should also be enrolled. It should be noted that there is often temporary, but unsustained postoperative improvement after any type of surgical manipulation of the spinal cord, potentially attributable to decompression, lysis of adhesions (detethering), or other unknown factors. Thus, a placebo group with no spinal cord manipulation provides only limited value as a control but, at a minimum, allows double blinding. Important to any SCI trial, including a chronic SCI study, is long-term assessment to ensure that any noted change is sustained. Finally, historical controls tend to be of limited value in any clinical trial. ${ }^{1}$

In summary, investigators in any SCI clinical trial are encouraged to always adhere to the highest investigative standards. Optimally, these standards should include the best possible control group and adoption of best practices for ensuring the highest standard of informed consent. In every Phase 2 clinical trial, assessments should include at least single-blinded and objective, reliable, and reproducible measures of study end points. Trials that do not incorporate control subjects, blinded assessments, and objective statistical analysis will lead to an inability at the conclusion of the trial to draw definitive conclusions regarding the potential benefit of a candidate therapy. There is a cost to the SCI community and society in conducting a trial that subjects people to risk, and that, at the end of the day, yields no useful conclusion.

Enrollment in trials of uncertain benefit and potential risk It is important that subjects solicited for enrollment into experimental clinical trials understand that they are 
consenting to a purely experimental procedure that is of no known benefit, which could cause serious adverse events including worsening of neurological function or death. Potential benefits of a clinical trial should not be exaggerated.

Research studies should proceed only if they are adequately designed to yield interpretable information regarding the objective benefit or lack of benefit of an experimental therapy. All clinical trials should be designed in a fashion to yield valid and useful information. Therefore, the trial should include appropriate control groups, objective data collection and analysis, blinded analysis (whenever possible), and extended assessments for a time period sufficient to draw clear conclusions.

An ethical issue arises when considering whether it is appropriate that a treatment not yet proven in adults should also be tested in children. Given that children are a particularly vulnerable population, a higher standard should be considered, where a study only includes children and if the investigator has evidence that the intervention is likely to be particularly effective or only effective in a younger population.

\section{Fetal tissue and stem cell research}

Clinical trials in SCI may wish to take into account that research involving human embryonic material, including stem cells, is an ongoing topic of ethical discussion in society. Researchers should ensure that both the written and verbal components of the informed consent process are clear about the nature of the cellular material being used.

\section{Clinical trials in developing countries}

Patients undergoing experimental interventions in developing countries should be aware that regulatory and ethical standards may differ from their home country. Nevertheless, any human clinical trial must minimally adhere to guidelines of the Declaration of Helsinki, the Belmont Report, and the standards of the host country. Where these standards come into conflict, it is the responsibility of the investigators to work with review committees and regulatory agencies to determine how best, if at all, the study can proceed.

\section{Competition for human subjects}

As several novel interventions transition to SCI clinical trials, there may be competition for enrollment of human subjects, as the total pool of acutely injured patients is relatively small. Investigators should therefore design trials to enroll a sufficient number of subjects to yield interpretable data, avoiding over-enrollment. The involvement of well-qualified statisticians in the development of a clinical study (including adaptive trial design) is important from both an ethical and efficiency standpoint.

\section{Other topics in trial recruitment and design}

\section{Surgical standardization}

As noted above, lack of standardization in the 'routine' surgical management of SCI constitutes an uncontrolled variable in clinical trials. This fact should be recognized and consideration be given to standardize surgical therapy within an individual trial. At a minimum, these practices should be recorded in all trials.

Similarly, practices of post-injury traction for anatomic reduction vary among centers, constituting an uncontrolled variable. Consideration should be given to standardize traction protocols within individual trials. At a minimum, these practices should be recorded in all trials.

\section{Rehabilitation standardization}

Also as noted previously, programs of rehabilitation constitute an uncontrolled variable that may affect outcomes in SCI trials. Programs of rehabilitation and funding for rehabilitation differ widely within and across nations. Consideration should be given to standardize rehabilitation protocols within individual trials. At a minimum, these practices should be recorded in all trials. $^{5}$

\section{Post-trial treatment for control subjects, and} compassionate use protocols

In trials using sham surgical controls, it has sometimes been the practice of study investigators to offer the option of undergoing active treatment at the conclusion of the study-monitoring period. Consideration could be given to the possibility that if a test substance meets primary efficacy outcomes at the end of the trial, placebo or sham surgery subjects could then be offered the therapy. Conversely, if a test substance is administered in a clinical trial and does not meet efficacy measures, whether subjects should be provided the option for therapy should be carefully assessed. Pragmatically, this would only be an option for a SCI trial targeting chronic stages of injury.

'Compassionate use' refers to the provision of a test article to subjects outside the context of a clinical trial, in 'special circumstances'. The compassionate use of experimental therapies undergoing clinical testing can undermine the objective assessment of efficacy, and has possible ethical implications for the good of the subject and society.

\section{Informed consent document guidelines}

Some individuals faced with the disability caused by SCI may choose to enter a study not because they have weighed the potential risks against a small chance of benefit, but because the desperation of their circumstance leads them to disregard anything other than the possibility of a benefit. This problem places a higher 
ethical obligation on researchers recruiting potential research subjects for a trial in SCI.

Informed consent is the centrepiece for the ethical conduct of research involving human subjects. An important part of that process is to clearly convey the probable and possible harms to someone who agrees to be a research subject. However, there are many other aspects that should be made clear as well. These include, but are not limited to, making clear that (1) an experimental intervention is research, not therapy, and the reason for conducting the study is because we do not yet know if it is safe and/or beneficial; (2) participation in the research study is not a prerequisite for receiving treatment that would otherwise have been available; and (3) participation is always voluntary, and the subject can withdraw at any time for any reason.

The following are examples of sample language that should be considered in informed consent documents.

\section{Risk of pain}

Therapies that aim to improve the growth of injured connections in the spinal cord could possibly also stimulate the growth of damaged pain fibers or sprouting from undamaged pathways, resulting in heightened pain that may be permanent or poorly responsive to therapy.

\section{Risk of spasticity, dysreflexia}

Therapies that aim to improve the growth of injured connections in the spinal cord could possibly also stimulate the growth of any fiber type in the spinal cord, resulting in an unknown spectrum of side effects including worsened spasticity or increased autonomic dysreflexia (a syndrome of elevated blood pressure that can be dangerous or fatal if untreated).

\section{Risk of loss of function}

There is a possibility that the experimental therapy will improve function, cause no change in function, or cause a minor or a major loss in function, possibly including segments of the spinal cord that are currently unaffected by injury.

\section{Uncertainty of adverse effects}

Because this is an experimental procedure, there may be risks that are currently unforeseeable. Any significant new findings regarding the potential of adverse events in this trial will be disclosed.

\section{Risk of infection}

Any invasive procedure carries the potential risk of infection, particularly implantation of cells that are not fully sterilized prior to implantation. Cellular therapies may also require the administration of immunosuppressive agents, rendering subjects less resistant to serious adverse effects such as pneumonia or urinary tract infections.

\section{Summary}

SCI is a challenging medical condition to study because of the many differences that exist between affected individuals. The ICCP Guidelines Panel strongly supports the use of appropriate, well-considered control groups to generate results that can provide clear interpretable answers regarding efficacy and safety of any experimental intervention.

\section{Glossary}

ASIA (American Spinal Injury Association) Classification (Grades) describes the completeness of a spinal injury. An individual with an ASIA A classification has no motor or sensory function at the level of S4-S5 sacral segments. ASIA B has some sensory function below the neurological level, including S4-S5, but has no motor function. ASIA $\mathrm{C}$ has some motor function below the neurological level, but more than half of the key muscles involved have a muscle grade score that is less than 3 . ASIA D has motor function below the neurological level, but more than half of the key muscles have a muscle grade of 3 or more. ASIA E indicates normal motor and sensory function.

Helsinki declaration was developed by the World Medical Association and is a set of ethical principles for the medical community regarding human experimentation. It was originally adopted in June 1964 and has since been amended multiple times. The recommendations concerning the guidance of physicians involved in medical research may be found at http://www.wma.net/ e/policy/b3.htm.

Belmont report is a report created by the former United States Department of Health, Education, and Welfare (which was renamed the Department of Health and Human Services) entitled 'Ethical Principles and Guidelines for the Protection of Human Subjects of Research.' The text is available at http://www.hhs.gov/ ohrp/humansubjects/guidance/belmont.htm.

Phase 1 clinical trial constitutes the first-stage of clinical testing in humans. Phase 1 trials assess the safety, tolerability, pharmacokinetics, pharmacodynamics, and maximum tolerated dose of a therapy, but is not large enough to assess efficacy.

Phase 2 clinical trial is an early, controlled clinical study conducted to obtain some preliminary (exploratory) data on the effectiveness or activity of the experimental therapy. This phase of testing also helps determine the common short-term side effects and risks associated with the drug. Phase 2 studies are typically well controlled, closely monitored, and conducted in a relatively small number of subjects.

Phase 3 clinical trial is a controlled trial on patient numbers sufficient to provide a definitive assessment of efficacy. If necessary, comparison is made to currently available alternative treatments.

Phase 4 clinical trial involves the post-marketing safety surveillance and studies to delineate additional information about the drug's risks, benefits, and optimal use. 


\section{References}

1 Lammertse D et al. Guidelines for the conduct of clinical trials for spinal cord injury (SCI) as developed by the ICCP Panel: clinical trial design. Spinal Cord 2007; 45: 232-242 (this issue).

2 Fawcett JW et al. Guidelines for the conduct of clinical trials for spinal cord injury (SCI) as developed by the ICCP Panel: spontaneous recovery after spinal cord injury and statistical power needed for therapeutic clinical trials. Spinal Cord 2007; 45: 190-205 (this issue).

3 Ellaway PH et al. Towards improved clinical and physiological assessments of recovery in spinal cord injury: a clinical initiative. Spinal Cord 2004; 42: 325-337.

4 Dietz V, Curt A. Neurological aspects of spinal cord repair: promises and challenges. Lancet Neurol 2006; 5: 688-694.

5 Steeves JD et al. Guidelines for the conduct of clinical trials for spinal cord injury (SCI) as developed by the ICCP Panel: clinical trial outcome measures. Spinal Cord 2007 45: 206-221 (this issue).

6 Fehlings MG, Perrin RG. The timing of surgical intervention in the treatment of spinal cord injury: a systematic review of recent clinical evidence. Spine 2006; 31: S28-S35.
7 Tator CH, Fehlings MG, Thorpe K, Taylor W. Current use and timing of spinal surgery for management of acute spinal surgery for management of acute spinal cord injury in North America: results of a retrospective multicenter study. J Neurosurg 1999; 91: 12-28.

8 Freeman TB et al. Use of placebo surgery in controlled trials of a cellular-based therapy for Parkinson's disease. $N$ Engl J Med 1999; 341: 988-992.

9 Rascol O. Assessing the risk of a necessary harm: placebo surgery in Parkinson disease. Neurology 2005; 65: 982-983.

10 The EC/IC Bypass Study Group. Failure of extracranialintracranial arterial bypass to reduce the risk of ischemic stroke. Results of an international randomized trial. $N$ Engl $J$ Med 1985; 313: 1191-1200.

11 Olanow CW et al. A double-blind controlled trial of bilateral fetal nigral transplantation in Parkinson's disease. Ann Neurol 2003; 54: 403-414.

12 Freed CR et al. Transplantation of embryonic dopamine neurons for severe Parkinson's disease. N Engl J Med 2001; 344: $710-719$. 